\title{
Survey on Recommendation System
}

\author{
Lipi Shah \\ Research Scholar \\ Dept. of Information \\ Technology \\ G.H Patel College of Engg. \& \\ Tech., \\ Gujarat, India.
}

\author{
Hetal Gaudani \\ Associate Professor \\ Dept. of Computer Engineering \\ G.H Patel College of Engg. \& \\ Tech., Gujarat, India.
}

\author{
Prem Balani \\ Assistant Professor \\ Dept. of Information \\ Technology \\ G.H Patel College of Engg. \& \\ Tech., Gujarat, India.
}

\begin{abstract}
This paper describes the overview of recommendation system. The recommendation system is the sub-part of the data mining field. This is the era of the e-commerce business. Recommender systems are used to assists the enterprise to implement one-to-one marketing strategies. These type of strategies offer several advantages like establishing the customer loyalty, increase the probability of cross-selling, fulfilling the customer need by presenting the items or products of customer interest. The recommendation system (RS) is crucial in many applications on the web. The recommendation system is mainly classified into following three categories: content-based, collaborative-based and hybrid approaches. Different categories have its own advantages as well as disadvantages. This paper describes the different techniques in each category and the issues in each category.
\end{abstract}

\section{Keywords}

Recommendation system, content based filtering, collaborative filtering, hybrid approach.

\section{INTRODUCTION}

Recommendation system is used different data mining techniques to generate meaningful suggestions to individual user or the group of users for items or products or elements that might interest them. This is the era of the web; lots of web data are available on the web. On the web , where the number of choices are overwhelming, so there is need of the information filtering on the web. Although many different approaches to recommender systems have been developed in past few years, the interest in this area is still high because growing demand of practical applications, which can deal with personalized recommendation and deal with large amount of overloaded data. By information filtering, one can prioritized the information and efficiently convey the relevant information to user and avoid the information overloading problem. As a user has large amount of choices from these overloaded information, user only know the some of his/her relevant information, rest of relevant information he/she don't know. So to navigate the users according to their taste or preference the recommendation system comes into the picture. The first recommendation system [21] is developed by Goldberg, Nichols, Oki \& Terry in 1992. Tapestry was an electronic messaging system, in which allowed the user to rate message the item (like or dislike) defined by the $\mathrm{M}$. Deshpande and G.Karypis: A personalized information filtering technology used to either predict whether a particular user will like a particular product or item (prediction problem) or to identify a set of $\mathrm{N}$ items products that will be of interest to a certain user. Further many other applications also used the recommendation system for different purposes like make more profit in industry, make an effective and efficient personalized result in the system. Today company has large amount of data available, which we can say raw data. To turn data tombs into "golden nuggets" of knowledge, intelligent recommendation system is used. Many of the company used this type of system like books, CD by amazon.com, CD by CDNOW, movies by movie lens etc to make more accurate prediction of user's mind. In this paper, section 2.describes the existing system, section 3.describes recommendation system approaches and their issues, section 4.describes comparisons of that approaches and section 5 give the conclusion.

\section{EXISTING SYSTEM}

The root of the recommendation system is the result of the extensive work in cognitive science [25], approximation theory [39], information retrieval [19], forecasting theories [35], and recommendation system emerged as an independent area in the mid-1990s. There are many real world applications available that use the recommendation system to help their users to find more appropriate products or items at users point of view and increase the production as well as profit at business point of view. In daily work everyone is using the Microsoft word is the one of the best example of RS, in which words are recommend to users. Another good example is mobile keypad, in which messaging application, user's type words are learned intelligently and in the future the wrong words are recommending by the correct words. Some of the real world examples are Amazon, movie lens, LIBRA, Pandora, Google etc. The amazon.com [15] item- to- item collaborative based filtering uses the traditional pure collaborative filtering, cluster models, search -based methods to recommend the same type of items to same group of users for books, CDs recommendation. Pandora [27][18] recommend the music, which is based on deep item analysis (Music genome project theory [33]) and user's preference represents as collection of items. Movie lens [23] recommended the movies based on the collaborative filtering, built the profile by asking the user's for movie preference (rating) and search for similar profile, also uses the stochastic and heuristic model to improve the profile matching. Netflix [13] is used for movie recommendation based on matrix factorization methods which is the one of the model based approach. As a time user's mind changes or interest may be change, Netflix has implemented this approach in the model based approach. Semantic based friend recommendation [32] uses the graph theory and page rank to recommend the top $n$ similar users to target user based on daily activity. Google [27] uses the page rank algorithm and page link structure to recommend the related web-pages to users and also uses the location, recent search activity of users and account past history to recommend the most suitable web-pages to target users. Based on the different application and different data available, different recommendation approaches are used by 
the system as discuss above. Each approach has its advantages and disadvantages which is discussed in the next section.

\section{RECOMMENDATION APPROACHES}

Recommendation system is the very big sub-domain of the data mining field. There are mainly two approaches in the personalized RS: Personalized and non-personalized. Each approach has different statistics and machine learning techniques. Non -personalized recommendation system in which system give same recommendation of items to all the users of the system. It doesn't interpret with each user's profile, rather than evaluate the system whole data at a onetime rather than individual user's data. But the here, each user's preference or interest is not considered, so whatever recommendation is given may or may not like by the users. This type of recommendation is used by the YouTube for most popular video by the aggregation method of the whole users like that video by average, mean etc methods .But all types of users may or may not like so the another approach the personalized recommendation system comes into the picture. Personalized recommendation system consider each user's preference or interest, so more effectively recommend particular items to user or group of the user's (community). There are mainly three approaches are in personalized recommendation system: content-based filtering, collaborative filtering and hybrid approach. Content-based filtering: Characteristics originate form information items. Collaborative filtering: Characteristics originate from user's environment (user's pattern, social etc) each of this approach has its advantage as well as disadvantage. So overcome these disadvantages, hybrid approach is used that is combination of both approach.

\subsection{Content-based filtering}

Content-based filtering (CBF) also known as "cognitive filtering", recommend the items based on the user's item profile and user profile. This type of profiles is created at the beginning, when the user creates the account and starts using the system. As a user more interaction with the system, more strong user profile is created. Here, only user's information is enough rather than other similar users, so very small scope of information are needed for recommendation.

The idea behind CBF system is that "if user like the item in the past, then user probably like similar type of item in future", so CBF compare the user's item profile with current item's profile and try to recommend the similar type of items that user may like. The profile of user is made of the different keywords, so simple way the CBF system match the keywords of highly rated item profile .To built the user profile, user's item preferences and user information are needed, which can be gather explicitly or implicitly. In the movie-lens dataset, user's profile is described by demographic information like gender, occupation, age, zip code etc. Explicit information can be gathered by personal input by clicking the checkbox, giving the start rating, thumbs up and down etc. But some time user may not give this type of information directly so need of the implicit data collection is needed. Thus system uses the implicit feedback to gather the information of user.

Content-based systems are designed mostly to recommend text-based items, so keywords are use as content here. For example, Fab system [8] which recommends Web pages to users, represents Web page content with the 100 most important words. Syskill \& Webert system [2] represents documents with the 128 most informative words. Different algorithms are available for content based filtering. The most commonly used techniques are TF-IDF, naïve bayes classifier etc.

\section{TF-IDF:}

It is a numerical statistic that is intended to reflect how important a word is to a document in a collection.TF-IDF (term frequency- inverse document frequency) is used to give weight to particular word in the information retrievals, which is as follow: [42] Assume that $\mathrm{N}$ is the total number of documents that can be recommended to users and that keyword $k_{j}$ appears in $n_{i}$ of them. Moreover, assume that $f_{i, j}$ is the number of times keyword $k_{i}$ appears in document $d_{i}$. So, the term frequency (or normalized frequency) $\mathrm{TF}_{\mathrm{i} \tilde{j} \mathrm{j}}$ of keyword $k_{i}$ in document $d_{j}$, is defined as:

$$
\mathrm{TF}_{\mathrm{i}_{\mathrm{j} j}}=\frac{\mathrm{f}_{\mathrm{i}, \mathrm{j}}}{\max _{\mathrm{z}} \mathrm{f}_{\mathrm{z}, \mathrm{j}}}
$$

while computing $\mathrm{TF}$, all terms are considered equally important. However, it is known that certain terms, such as "is", "of", and "that", may appear a lot of times but have little importance. Thus need to weigh down the frequent terms while scale up the rare ones, by computing the inverse document frequency $\left(I D F_{i}\right)$ for keyword $k_{i}$ is defined as:

$$
I D F_{i}=\log \frac{N}{n_{i}^{i}}
$$

then, the TF-IDF weight for keyword $k_{i}$ in document $d_{j}$ is defined as:

$$
w_{i, j}=T F_{i, j} \times I D F_{i}
$$

The content of document $d_{j}$ is defined as:

$$
\operatorname{Content}\left(d_{j}\right)=\left(w_{1 j}, \ldots, w_{k j}\right)
$$

\section{Naïve Bayes Classifier:}

Besides of the previous traditional heuristic approach, contentbased filtering can be done through Bayesian classifiers and various machine learning techniques, including clustering, decision trees and artificial neural networks [10]. Bayesian classifier is used to estimate the probability that a document is liked or not. Naïve Bayes classifier is the machine learning, family of simple probabilistic classifier based on applying Bayes' theorem with strong independence assumptions between the features. Naive Bayesian classifier is used to estimate the following probability that webpage $p_{j}$ belongs to a certain class $C_{i}$ (e.g., relevant or irrelevant) given the set of keywords $k_{1, j} ; \ldots ; k_{n, j}$ on that webpage as:

$$
P\left(C_{i} \mid k_{1 j} \& \ldots . . \& k_{n j}\right)
$$

Here assume that keywords are independent and, therefore, the above probability is proportional to:

$$
P\left(C_{i}\right)_{x}^{\Pi} P\left(k_{x, j} \mid C_{i}\right)
$$

Furthermore, both $\mathrm{P}\left(k_{x, i} \mid C_{i}\right)$ and $\mathrm{P}\left(C_{i}\right)$ can be estimated from the underlying training data. Therefore, for each page $p_{j}$, the probability $\mathrm{P}\left(\mathrm{Ci} \mid \mathrm{k}_{1, \mathrm{j}} \& \ldots \& x_{n, i}\right)$ is computed for each class 
$C_{i}$ and page $p_{j}$ is assigned to class $C_{i}$ having the highest probability[10].

Other some techniques are used in text mining are adaptive filtering [6][29], which focuses on becoming more accurate at identifying relevant documents incrementally by observing the documents one-by-one in a continuous document stream, threshold setting [1][36], which focuses on determining the extent to which documents should match a given query in order to be relevant to the user.

Using CBF only particular target user information needed rather than similar type of user's information so, there will not be cold start problem. Another advantage is that, if user with unique taste or preference then also it will recommend the user's preference items and also there will not be first rater problem that new item arrives that is not rated by many users. Although there are many advantages, there are some drawbacks are also there.

\subsubsection{Limitation of content-based filtering 1. New user problem:}

It is also known as the lack of information problem. When the new user enter in the system, system doesn't have sufficient information of user profile and his /her preference for particular products, so proper item profile cannot created based on that So as a result poor recommendation can be done.

\section{Overspecialization:}

Recommendation system only recommends the items or product that user has liked or rated the highly in the past. Based on the past data available, system recommends similar type of the items or products. System does not recommend these items that are different from anything that the user has seen before. Sometimes this might become problem because the user might want to try something new and the system would never make it happen. For ex. "If user has liked all comedy type of movie in the past, then system only recommend other comedy movies that user may not seen yet, but user may like fiction movies also but system never recommend." So, overspecialization make the scope very small, though there are many other items that user likes. The use of genetic algorithms has been proposed as a possible solution in the context of information filtering [12]. Another solution is that doesn't recommend the too much similar items to user based on set some thresholding value.

\subsection{Collaborative filtering}

Collaborative filtering (CF) is liked-minded user recommendation approach. In CF systems a user is recommended items based on the past ratings of all users collectively. Collaborative Filtering (CF) systems work by collecting user feedback in the form of ratings for items in a given domain and exploiting similarities in rating behavior amongst several users in determining how to recommend an item. Grundy system [41] was

the first recommender system, which proposed using stereotypes as a mechanism for building models of users based on a limited amount of information on each individual user. Using stereotypes, system creates individual user's model is and relevant books are recommend to each user. Video Recommender [4], amazon.com [15] for books recommendation, Jester system that recommends jokes [37], Group Lens [9],[40], are the some of the examples of collaborative filtering.
CF methods can be further sub-divided into neighborhoodbased and model-based approaches. Neighborhood-based methods are also commonly referred to as memory based approach.

\subsubsection{Memory-based approach}

In this approach, heuristics make the rating prediction, based on the all the users previous data (Entire collection of rated items by users previously). User's preferences are made by the calculating the algorithm results again and again as the user query. Here no any pre-compute data is there. The unknown rating $r_{C_{s} s}$ for user $\mathrm{c}$ and item $\mathrm{s}$ is usually computed as an aggregate of the ratings of some other (usually, the $\mathrm{N}$ most similar) users for the same item $\mathrm{s}$ as follows:

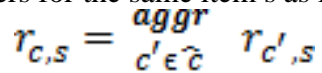

(7) Where $\hat{c}$ denotes the set of $\mathrm{N}$ users that are the most similar to user $\mathrm{c}$ and who have rated item $\mathrm{s}(\mathrm{N}$ can range anywhere from 1 to the number of all users).

Some of the aggregate functions are the absolute average of rating of similar users, weighted multiplicative rating sum of the similar users etc

Memory based approach further divided into two types: UserUser collaborative filtering and Item-Item collaborative filtering.

Both approaches use the $\mathrm{KNN}$ (K-nearest neighbor) rule. Steps for KNN rule is as follow:

1. $\mathrm{X}$ is the target customer.

2. Chose the value of the $\mathrm{K}$. $(\mathrm{k}=$ number of nearest users).

3. Find the similarity of the $\mathrm{K}$ users with $\mathrm{X}$, by means of the similarity measurement techniques. Ex. Euclidean distance, jaccards similarity, cosine similarity etc.

$$
\text { Euclidean }=\sqrt{\sum_{\mathrm{i}=1}^{\mathrm{k}}\left(\mathrm{x}_{\mathrm{i}}-\mathrm{y}_{\mathrm{i}}\right)^{2}}
$$

4. Chose the weight function and find the single real number.

User-User collaborative filtering and Item-Item collaborative filtering are described below:

\section{User-user collaborative filtering}

This approach was proposed in the end of 1990s by the professor of University of Minnesota Jonathan L.Herlocker. In this filtering, subset of users is chosen based on the similarity to the active users. After that weighted combination of their rating is used to predict of rating for the user. The generalized steps are as follow:

1. Assign a weight (similarity) to all users with respect to similarity with the active user.

2. Select the value of the $\mathrm{k}$, so can find $\mathrm{k}$-like minded user.

3. Compute the prediction for the target user based on the weight function and k-similar user's rating.

In the step 1 , the weight $w_{a, u}$ computes the similarity between the user $u$ and the active user a. The most common method is Pearson correlation coefficient to measure the similarity 
between two users defined as following equation [28]:

$$
w_{a, u}=\frac{\sum_{i \in I}\left(r_{a, i}-\overline{r_{a}}\right)\left(r_{u, i}-\overline{r_{u}}\right)}{\sqrt{\sum_{i \in I}\left(r_{a, i}-\overline{r_{a}}\right)^{2} \sum_{i \in I}\left(r_{u, i}-\overline{r_{u}}\right)^{2}}}
$$

Where $\mathrm{I}$ is the set of items rated by both users, $r_{u, i}$ is the rating given to item $\mathrm{i}$ by user $\mathrm{u}$, and $r_{u}$ is the mean rating given by user $u$.

In step 2, randomly value of $\mathrm{k}$ is decided based on the evaluation method.

In step 3, final prediction is computed as the weighted average of deviations from the neighbor's mean as follows:

$$
\mathrm{P}_{\mathrm{a}, \mathrm{i}}=\overline{\mathrm{r}_{\mathrm{a}}}+\frac{\sum_{\mathrm{u} \in K\left(\mathrm{r}_{\mathrm{u}, \mathrm{i}}-\overline{\mathrm{r}_{\mathrm{u}}}\right) \times w_{\mathrm{au}}}}{\sum_{\mathrm{u} \in \mathrm{K}^{\mathrm{w}}} \mathrm{w}_{\mathrm{au}}}
$$

Where $p_{a, i}$ is the prediction for the active user a , for item i, $w_{a, u}$ is the similarity between users a and $\mathrm{u}$, and $\mathrm{K}$ is the neighborhood or set of most similar users.

\section{Item-item collaborative filtering}

This approach was proposed by the researchers of University of Minnesota in 2001 [20]. As a system grows high, number of users increase so, conventional neighbor base CF doesn't scale well because the complexity of finding of similar users grow high. So new approach was proposed [15] of item-item collaborative filtering that is rather than finding similar users, find similar items.

In these approach similarities between pairs of items $i$ and $j$ are computed offline using Pearson correlation as:

$w_{a, u}=\frac{\sum_{u \in U}\left(r_{u, i}-\overline{r_{i}}\right)\left(r_{u, j}-\overline{r_{j}}\right)}{\sqrt{\sum_{u \in U}\left(r_{u, i}-\overline{r_{i}}\right)^{2}} \sqrt{\sum_{u \in U}\left(r_{u, j}-\overline{r_{j}}\right)^{2}}}$

Where $\mathrm{U}$ is the set of all users who have rated both items $i$ and $\mathrm{j}, r_{u, i}$ is the rating of user $\mathrm{u}$ on item $\mathrm{i}$, and $\overline{r_{i}}$ is the average rating of the $i^{\text {th }}$ item across users.

the rating for item $i$ for user a can be predicted using a simple weighted average, as:

$$
\mathrm{P}_{\mathrm{a}, \mathrm{i}}=\frac{\sum_{\mathrm{j} \in \mathrm{K}} \mathrm{K}_{\mathrm{a} j \mathrm{j}} \mathrm{w}_{\mathrm{i}, \mathrm{j}}}{\sum_{\mathrm{j} \in \mathrm{K}} \mid \mathrm{w}_{\mathrm{ij}} \mathrm{j}}
$$

Where $\mathrm{K}$ is the neighborhood set of the $\mathrm{k}$ items rated by a, that are most similar to $i$.

Other alternative techniques for this approach are Significance Weighting [3], default voting [34], Inverse User Frequency [34].

\subsubsection{Model-based approach}

In contrast to memory-based approach, recommend user preference by estimating parameters of statistical models for user ratings. Unlike neighborhood based methods that generate recommendations based on statistical notions of similarity between users or items, Latent Factor models assume that the similarity between users and items is simultaneously induced by some hidden lower-dimensional structure in the data. Here one pre-computed model is design based on the available data. When the user query appear this model based approach give fast answer to the user's preference. Based on the dependency in the data, this approach reduces the dimension. So, this approach reduces the memory and reduces the processing time. Through this approach system can visualized more accurately and reduce the error also. Different methods are available to find hidden (latent) features. Most commonly used methods are MF (Matrix factorization), SVD (Singular value decomposition). MF (Matrix factorization)

Matrix factorization is a dimensionality reduction technique that factorizes a matrix into a product of matrices, usually two. It is used to fill the sparse matrix by the pre-computed model. User u's rating of item $\mathrm{i}$, which is denoted by $r_{u, i}$, leading to the estimate as follows [13]: $\bar{r}_{u i}=q_{i}^{T} p_{u}$

Here, for a given item $i$, the elements of $q_{i}$ measure the extent to which the item possesses those factors, positive or negative. For a given user $u$, the elements of $p_{u}$ measure the extent of interest the user has in items that are high on the corresponding factors, again, positive or negative. To learn the factor vectors $\left(p_{u}\right.$ and $\left.q_{i}\right)$, the system minimizes the regularized squared error on the set of known ratings as follows:

$$
{ }_{q^{*} \cdot p^{*}}^{\min } \sum_{(u, i) \in k}\left(r_{u, i}-q_{i}^{T} p_{u}\right)^{2}+\lambda\left(\left\|q_{i}\right\|^{2}+\left\|p_{u i}\right\|^{2}\right)
$$

Here, ${ }^{\lambda}$ is regularization parameter and $\kappa$ is the set of the $(u$, I) pairs for which $r_{u, i}$ is known.

Using gradient descent and alternative least square method they add user and item bias to MF and make more accurate model.

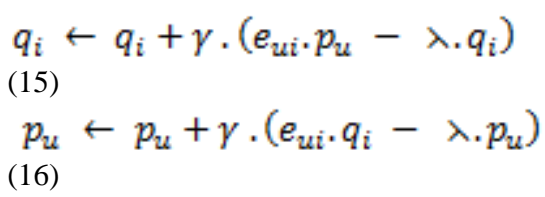

$\gamma$ is the learning step and through this error can be minimized as:

$$
e_{u i} \stackrel{\text { def }}{=} r_{u_{i} i}-q_{i}^{T} p_{u}
$$

Every time loop interacted by equation (15) (16) and try to minimized error by equation (17).

Other collaborative filtering methods include a Bayesian model [14], a probabilistic relational model [11], a linear regression [31], and a maximum entropy model [7], sequential decision problem and propose using Markov decision processes (a well-known stochastic technique for modeling sequential decisions) for generating recommendations.

\subsubsection{Limitation of collaborative filtering 1.Cold-start problem:}

There needs to be enough other users already in the system to find a match. Collaborative filtering is totally depends on similar neighbor in the system, but if these similar neighbors are not available in the system in the initial phase that is known as "cold-start" problem. This problem can be avoided by the hybrid approach. 


\section{First-rater problem:}

The system can not recommend an item that has not been previously rated. As new item entered in the system, not much users have referred that item, so not much rating of these items are available. So this is known as the "first rater problem". This can be removed by mixture of content-based filtering as a hybrid approach.

\section{Sparsity:}

If there are many items to be recommended, even if there are many users, the user/ratings matrix is sparse, and it is hard to find users that have rated the same items. In online shops that have a huge amount of users and items there are almost always users that have rated just a few items. Using collaborative and other approaches recommender systems generally create neighborhoods of users using their profiles. If a user has evaluated just few items then its pretty difficult to determine his taste and he/she could be related to the wrong neighborhood. Sparsity is the problem of lack of information [20].

\section{Popularity Bias:}

System can not recommend items to someone with unique tastes. Sometime the user has unique taste than all other users in the system, that problem is known as "popularity bias" problem. This can be solved by the hybrid approach by using content-based filtering over collaborative-filtering.

\subsection{Hybrid approach}

For better results some recommender systems combine different techniques of collaborative approaches and content based approaches.

By hybrid approach, limitation of content-based and collaborative approach can be avoided. Combination of these two approaches is done in the different ways can be classified as: $\quad 1$. Implementing collaborative and content-based methods separately and combining their predictions. 2. Incorporating some content-based characteristics into a collaborative approach.

3. Incorporating some collaborative characteristics into content-based approach.

4. Construct

general unified model that incorporate with both approach content as well a collaborative.

Different hybridization methods are described below:

\section{Weighted hybridization:}

Weighted hybridization combines the results of different recommenders content as well as collaborative, to generate a recommendation prediction by integrating the scores from each of the techniques in use by a linear formula. Initially weight of the each recommendation approach is given same. Afterwards, using different evaluation, the weight are adjusted as per need. The example of such type of system is Ptango[22].

\section{Switching hybridization:}

The system switches between recommendation techniques depending on a heuristic reflecting the recommender ability to produce a good rating prediction. So this type of system avoids the disadvantages of single type of approach by swapping between different approaches. The example of such type of system is daily learner [26] that used both content and collaborative approach in the switching manner.

\section{Mixed hybridization:}

Mixed hybrids combine recommendation results of different recommendation techniques at the same time instead of having just one recommendation per item. PTV system [38] is the example of mixed hybridization approach. It uses contentbased techniques based on textual descriptions of TV shows and collaborative information about the preferences of other similar types of users. Using mixed hybridization, it avoids "new item problem" by content-based filtering over the collaborative filtering. The content-based component can be relied on to recommend new shows on the basis of their descriptions even if they have not been rated by anyone.

\section{Cascade hybridization:}

In this hybridization, the recommendations of one technique are refined by another recommendation technique. The restaurant recommender EntreeC [5] is the example of the cascade hybridization. The cascade hybridization technique applies an iterative refinement process in constructing an order of preference among different items. In Entree, it uses its knowledge of restaurants to make recommendations based on the user's stated interests or preferences. The recommendations are placed in buckets of equal preference, and the collaborative technique is employed to break ties, further ranking the suggestions in each bucket.

\section{Feature Augmentation:}

In this hybridization, output from one technique is used as an input feature to another. The Libra system [28] makes content-based recommendation of books on data found in Amazon.com by employing a naive Bayes text classifier.

\section{Feature-combination:}

In this hybridization, Features from different recommendation data sources are thrown together into a single recommendation algorithm. The advantage of this technique is that, it does not always exclusively rely on the collaborative data. The example of this type of approach is Pipper [24] that used the collaborative filter's ratings in a content-based system as a feature for recommending movies.

\section{Meta-level:}

In this hybridization, the model learned by one recommender is used as input to another. The benefit of the meta-level method, especially for the content/collaborative hybrid is that the learned model is a précised representation of a user's interest, and a collaborative mechanism that follows can operate on this information-dense representation more easily than on raw rating data. It avoids the sparsity problem by using the entire model learned by the first technique as input for the second technique. LaboUr [17] is the example of this type of approach, in which instant-based learning to create content-based user profile that is then compared in a collaborative approach.

\section{MEASURES}

$$
\begin{aligned}
& \text { 1. Precision } \mathrm{P} \text { of a system is computed as: } \\
& \mathrm{P}=\frac{\text { \# correct answers provided }}{\text { \# answers provided }}
\end{aligned}
$$

Precision determines how good the answers are given.

2. Recall $R$ is calculated as:

$\mathrm{R}=\frac{\text { \# correct answers provided }}{\text { \# total answers to provide }}$

According to the above definitions , $\mathrm{R} \leq \mathrm{P}$. When coverage is $100 \%, \mathrm{P}=\mathrm{R}$.

Recall is also referred to as accuracy. 
3. F1-measure or balanced F-score, measure which determines the weighted harmonic mean of precision and recall, is defined as:

$\mathrm{F} 1=\frac{2 \mathrm{PR}}{(\mathrm{P}+\mathrm{R})}$

4. MAE (Mean absolute error) the average magnitude of errors in the forecast, without consider their direction.

$\operatorname{MAE}=\frac{\Sigma_{[\mathrm{u} i \mathrm{i}\}}\left|\mathrm{p}_{\mathrm{u}, \mathrm{i}}-\mathrm{r}_{\mathrm{u}, \mathrm{i}}\right|}{\mathrm{N}}$

Here $\mathrm{N}$ is the total number of the training set. $\mathrm{P}$ represents predicted answer and $\mathrm{r}$ represented actual answer.

5. RMSE is quadratic scoring rule which measures the average magnitude of the error. RMSE can be calculated as defined below:

RMSE $=\sqrt{\frac{1}{|T|} \underset{\text { (urr)eT }}{\Sigma}\left(s_{u r}-\hat{s}_{u r}\right)^{2}}$ (22)

Here, $\mathrm{T}$ is size of the training dataset. $s_{u, r}$ is the true value and $\hat{s}_{u, r}$ is the calculated value.

\section{COMPARISION}

There are different approaches in RS. Each of the approach has advantages and because of some disadvantages, some new approaches are invented to overcome that disadvantages. In the content-based approach, there are some problem like new user problem and overspecialization. So, to overcome these disadvantages, new approach comes into the picture that is collaborative filtering. Using collaborative filtering, it remove the disadvantages of content-based filtering. But, still there is problem in it like cold start problem, Sparsity, Popularity bias, first rater problem etc. So, to overcome new approach was invented that is hybrid approach. Each has their advantages as well as disadvantages which are describe below in table 1 .

Table 1.Comparison of RS approaches

\begin{tabular}{|c|c|c|}
\hline Approach & Advantage & Disadvantage \\
\hline $\begin{array}{c}\text { Content- } \\
\text { Based } \\
\text { Approach }\end{array}$ & $\begin{array}{c}\text { User with unique } \\
\text { taste can be } \\
\text { recommended } \\
\text { preference items. } \\
\text { Quality of system } \\
\text { improves over a } \\
\text { time. }\end{array}$ & -New user problem, \\
Overspecialization \\
Collaborative & $\begin{array}{c}\text { Overspecialization } \\
\text { problem can be } \\
\text { removed by finding } \\
\text { similar uses in the } \\
\text { system. Quality of } \\
\text { system improves } \\
\text { over a time. }\end{array}$ & -Cold start problem \\
& -Popularity bias \\
& \\
\hline
\end{tabular}

To overcome each another disadvantages, hybrid approach is used to improve the efficiency of recommendation system which is used both content-based and collaborative filtering on particular order to make system more efficient.

\section{CONCLUSION}

Recommendation system is the progressive field in the last decade, when numbers of content-based, collaborative and hybrid approaches are proposed for "enterprise growth and increase the productivity" as a part of industrial strength. Recommender systems open new opportunities of retrieving personalized information on the web. This paper discussed various real-world existing recommendation system which the different three recommendation approaches. Also discuss their strength as well as limitation with the diverse kind of hybridization strategies used to improve the performance. We also discuss the various learning algorithm used in generating recommendation model and evaluation method used in measuring quality and performance of the recommendation algorithm. We hope that issues presented in this paper will be the discussion for the next generation of recommendation technologies.

\section{REFERENCES}

[1] S. Robertson and S. Walker, "Threshold Setting in Adaptive Filtering," J. Documentation, vol. 56, pp. 312 331, 2000.

[2] M. Pazzani and D. Billsus, "Learning and Revising User Profiles The Identification of Interesting Web Sites," Machine Learning, vol. 27, pp. 313-331, 1997.

[3] J. Herlocker, J. Konstan, A. Borchers, and J. Riedl. An algorithmic framework for performing collaborative filtering. In Proceedings of 22nd International ACM SIGIR Conference on Research and Development in Information Retrieval, pages 230-237, Berkeley, CA, 1999. ACM Press.

[4] W. Hill, L. Stead, M. Rosenstein, and G. Furnas, "Recommending and Evaluating Choices in a Virtual Community of Use," Proc. Conf. Human Factors in Computing Systems, 1995.

[5] Burke R. Hybrid recommender systems: survey and experiments. User Model User-adapted Interact 2002;(12)

[6] 331-70

[7] G. Somlo and A. Howe, "Adaptive Lightweight Text Filtering," Proc. Fourth Int'l Symp. Intelligent Data Analysis, 2001

[8] D. Pavlov and D. Pennock, "A Maximum Entropy Approach to Collaborative Filtering in Dynamic, Sparse, High-Dimensional Domains," Proc. 16th Ann. Conf. Neural Information Processing Systems (NIPS '02), 2002.

[9] M. Balabanovic and Y. Shoham, "Fab: Content-Based, Collaborative Recommendation," Comm. ACM, vol. 40, no. 3, pp. 66-72, 1997.

[10] J.A. Konstan, B.N. Miller, D. Maltz, J.L. Herlocker, L.R. Gordon, and J. Riedl, "GroupLens: Applying Collaborative Filtering toUsenet News," Comm. ACM, vol. 40, no. 3, pp. 77-87, 1997.

[11] M. Pazzani and D. Billsus, "Learning and Revising User Profiles:The Identification of Interesting Web Sites," Machine Learning,vol. 27, pp. 313-331, 1997. 
[12] L. Getoor and M. Sahami, "Using Probabilistic Relational Models for Collaborative Filtering," Proc. Workshop Web Usage Analysis and User Profiling (WEBKDD '99), Aug. 1999.

[13] B. Sheth and P. Maes, "Evolving Agents for Personalized Information Filtering," Proc. Ninth IEEE Conf. Artificial Intelligence for Applications, 1993

[14] Koren, Y., Bell, R.M., Volinsky, C.: Matrix factorization techniques for recommender systems, Computer (2009).

[15] Y.-H. Chien and E.I. George, "A Bayesian Model for Collaborative Filtering," Proc. Seventh Int'l Workshop Artificial Intelligence and Statistics, 1999.

[16] Greg Linden, Brent Smith, and Jeremy,"Amazon.com Recommendations Item-to-Item Collaborative Filtering ",

[17] IEEE (2003)

[18] Paul Resnick, Neophytos Iacovou, Mitesh Suchak, Peter Bergstrom, John Riedl, GroupLens: an open architecture for collaborative filtering of netnews, Proceedings of the 1994 ACM conference on Computer supported cooperative work, p.175-186, October 22-26, 1994, Chapel Hill, North Carolina, United States

[19] Schwab I, Kobsa A, Koychev I. Learning user interests through positive examples using content analysis and collaborative filtering. Draft from Fraunhofer Institute for Applied Information Technology, Germany; 2001.

[20] Iskold, A., Rethinking Recommendation Engines,http://alexiskold.wordpress.com/2008/02/25/reth inking-recommendation-engines/ ， 25 February 2008, Rethinking Recommendation Engines.

[21] G. Salton, Automatic Text Processing. Addison-Wesley, 1989

[22] Pronk, V., Verhaegh, W., Proidl, A., and Tiemann, M., Incorporating user control into recommender systems based on naive bayesian classification. In RecSys '07: Proceedings of the 2007 ACM conference on Recommender systems, pages 73-80,2007

[23] Witten I. H. and Frank I. Data Mining, Morgan Kaufman Publishers, an Francisco, 2000.

[24] Claypool M, Gokhale A, Miranda T, Murnikov P, Netes D, Sartin M. Combining content- based and collaborative filters in an online newspaper. In: Proceedings of ACM SIGIR workshop on recommender systems: algorithms and evaluation, Berkeley, California; 1999.

[25] Ujjin, S.,Bentley,P.J., Building a Lifestyle Recommender System, Poster Proceedings of the 10th International World, 2001

[26] Basu C, Hirsh H, Cohen W. Recommendation as classification: using social and content-based information in recommendation. In: Proceedings of the 15th national conference on artificial intelligence, Madison, WI; 1998. p. $714-20$.

[27] E. Rich, "User Modeling via Stereotypes," Cognitive Science, vol. 3, no. 4, pp. 329-354, 1979.

[28] Billsus D, Pazzani MJ. A hybrid user model for news story classification. In: Kay J, editor. In: Proceedings of the seventh international conference on user modeling, Banff, Canada. Springer-Verlag, New York; 1999. p. 99 108.
[29] MacManus, R., A guide to Recommender Systems,http://www.readwriteweb.com/archives/recomm endation_systems_where_we_need_to_go.php , 26 january 2009 A guide to Recommender Systems

[30] Mooney RJ, Roy L. Content-based book recommending using learning for text categorization. In: Proceedings of the fifth ACM conference on digital libraries. ACM; 2000. p. 195-204.

[31] Y. Zhang, J. Callan, and T. Minka, "Novelty and Redundancy Detection in Adaptive Filtering," Proc. 25th Ann. Int'1 ACM SIGIR Conf., pp. 81-88, 2002.

[32] John S. Breese, David Heckerman and Carl Kadie. Empirical analysis of predictive algorithms for collaborative filtering. In Proceedings of the Fourteenth Annual Conference on Uncertaintyin Artificial Intelligence, pages 43-52, July 1998.

[33] B. Sarwar, G. Karypis, J. Konstan, and J. Riedl, "ItemBased Collaborative Filtering Recommendation Algorithm," Proc. 10 ${ }^{\text {th }}$ Int'l WWW conf, 2001.

[34] Zhibo Wang, Jilong Liao, Qing Cao, Hairong Qi, and Zhi Wang, "Friendbook: A Semantic-based Friend Recommendation System for Social Networks" IEEE TRANSACTIONS ON MOBILE COMPUTING,VOL. 13, NO. 99, MAY2014.

[35] Wintergreen, T., The Music Genome Project ${ }^{\circledR}$, http://www.pandora.com/mgp.shtml, 2000

[36] John S. Breese, David Heckerman, and Carl Kadie. Empirical analysis of predictive algorithms for collaborative filtering. In Proceedings of the Fourteenth Conference on Uncertainty in Artificial Intelligence, Madison, WI, July 1998

[37] J.S. Armstrong, Principles of Forecasting-A Handbook for Researchers and Partitioners. Kluwer Academic, 2001.

[38] Y. Zhang and J. Callan, "Maximum Likelihood Estimation for Filtering Thresholds," Proc. 24th Ann. Int'l ACM SIGIR Conf., 2001.

[39] K. Goldberg, T. Roeder, D. Gupta, and C. Perkins, "Eigentaste: A Constant Time Collaborative Filtering Algorithm," Information Retrieval J., vol. 4, no. 2, pp. 133-151, July 2001.

[40] Smyth B, Cotter P. A personalized TV listings service for the digital TV age. J Knowl-Based Syst 2000;13(23):53 9.

[41] M.J.D. Powell, Approximation Theory and Methods. Cambridge Univ. Press, 1981.

[42] P. Resnick, N. Iakovou, M. Sushak, P. Bergstrom, and J. Riedl, "GroupLens: An Open Architecture for Collaborative Filtering of Netnews," Proc. 1994 Computer Supported Cooperative Work Conf., 1994.

[43] E. Rich, "User Modeling via Stereotypes," Cognitive Science, vol. 3, no. 4, pp. 329-354, 1979.

[44] G. Salton, Automatic Text Processing. Addison-Wesley, 1989. 